\title{
Myocarditis in a 47-Year-Old Man with Myasthenia Gravis and Malignant Thymoma Following the Administration of a Tyrosine Kinase/CDK Inhibitor
}

\author{
Conor McDonald ${ }^{1,2 *}$, Jonathan Wackett ${ }^{1,2}$, Steve Philpot ${ }^{1,2}$ and Benjamin M Brady ${ }^{3,4}$ \\ ${ }^{1}$ Monash University, Melbourne, Australia \\ ${ }^{2}$ Intensive Care Department, Cabrini Hospital, Melbourne, Australia \\ ${ }^{3}$ Department of Hematology and Oncology, Cabrini Hospital, Melbourne, Australia \\ ${ }^{4}$ Peter McCallum Cancer Centre, Melbourne, Australia
}

Submission: May 28, 2020; Published: June 10, 2020

*Corresponding author: Conor McDonald, Monash University, Melbourne, Australia

\begin{abstract}
Summary
A 47-year-old man was admitted to an intensive care unit for increasing respiratory distress, persistent diarrhoea and general decline on a background of longstanding myasthenia gravis and metastatic thymic carcinoma. He had recently been involved in a phase 1 clinical trial with the drug FN-1501- an inhibitor of cyclin dependent kinases CDK2/CDK4/CDK6 and tyrosine kinase (TK) FLT3. The patient was found to have severe systolic dysfunction on echocardiography, consistent with myocarditis, for which he required inotropic support. It was only partially responsive to steroid treatment. In combination with the patient's myasthenia gravis and deconditioning, the myocarditis contributed to the patient's continued decline and ultimate death. This case explores the possibility that the patient's myocardial injury and subsequent death was related to the trial drug FN-1501. An association between TK inhibitors and cardiotoxicity has been outlined in the past but the cardiac effects of CDK inhibitors is still in question. There is no data presently implicating FN-1501.
\end{abstract}

Keywords: Myasthenia Gravis; Thymoma; Myocarditis; CDK/TK inhibitor; End of Life Care

Abbreviations: MG: Myasthenia Gravis; CDK: Cyclin Dependent Kinase; TK: Tyrosine Kinase; FLT3: Fms-like tyrosine kinase 3; CT: Computed Tomography; PET: Positron Emission Tomography; LV: Left Ventricular; CRP: C-Reactive Protein; WCC: White Cell Count; FEV1: Forced Expiratory Volume (1 second); FVC: Forced Vital Capacity; PEF: Peak Expiratory Flow; GIST: Gastro-Intestinal Stromal Tumor

\section{Background}

Myasthenia Gravis (MG) is a disease of the neuromuscular junction that is characterized by weakness and fatigability of skeletal muscles. Autoimmune antibodies are produced to the acetylcholine receptors, destroying a portion of them, and blocking their ability to produce action potentials.

The pillars of treatment include:

1.Therapy with anticholinesterases to increase available acetylcholine

2. Immunosuppression to subdue antibody producing B cells and

3. Thymectomy as over $35 \%$ of MG patients have thymic abnormalities and removal of the thymus can be associated with decreased disease burden [1].
Thymomas are epithelial tumors that have malignant potential. The key prognostic factor is whether they are localized or invasive, and around $50 \%$ of thymomas are invasive on presentation. Around $30 \%$ of patients with thymomas also have MG and up to $15 \%$ of patients with MG present with thymomas [2]. Therapy normally involves of resection and can include radiotherapy and chemotherapy. Common paraneoplastic syndromes associated with thymomas include myasthenia gravis and myositis [3]. Cyclin dependent kinases (CDK) are important signaling molecules for some cancers [4]. Tyrosine kinases are enzymes involved in the signaling cascades that regulate cell functions, such as cell division. The drug FN-1501 is a CDK2/CDK4/CDK6 and FLT3 (a tyrosine kinase receptor) inhibitor [5], thus it aims to interrupt 


\section{Cancer Therapy \& Oncology International Journal}

a cancer's signaling pathways. FN-1501 is currently in a phase 1 trial investigating its safety and effectiveness in treating advanced solid tumors. The potential for certain tyrosine kinase inhibitors to cause myocardial injury has been outlined in the past $[6,7]$, although there is nothing in the literature specifically implicating CDK/FLT3 inhibitors such as FN-1501.

\section{Case Presentation}

A 47-year-old male was admitted to an intensive care unit for increasing respiratory distress (with CPAP dependence), malaise, decreased appetite, persistent diarrhea, and general decline on a background of longstanding myasthenia gravis and metastatic thymic carcinoma. He had recently been involved in a phase 1 clinical trial with the drug FN-1501 to try and manage his thymic cancer and control myasthenia symptoms. Initially, his presentation was linked to myasthenia related myopathy with a possible superimposed infection and he was admitted into an HDU bed for monitoring and further tests. He had been entered into the trial following a recurrence of his thymic carcinoma first noted on chest CT which revealed a pleural thickening and adjacent mass that measured $7.1 \times 3 \mathrm{~cm}$ in the left lower lobe. A PET scan found moderate uptake in this area and that the mass was likely a metastasis of his thymic carcinoma

The patient had been admitted to the same ICU 6 weeks earlier for an invasive aspergillosis infection requiring several days of intubation for hemoptysis, mechanical ventilation, and treatment with voriconazole (subsequently changed to Posaconazole). Preliminary investigations yielded mixed results with abnormalities detected in the following areas:

- $\quad$ Blood count: $\mathrm{Hb} 106 \mathrm{~g} / \mathrm{L}$ (normal =130-150g/L)

- Renal function: Creatinine: $149 \mathrm{mmol} / \mathrm{L}$, Urea: $12 \mathrm{mmol} / \mathrm{L}$

- Inflammatory markers: CRP:49mg/L, WCC normal

- Bedside lung function tests: FEV1: 0.68L, FVC:0.70L, FEV1/FVC: 98\%, PEF: 116 l/min),

- Venous blood gas: $\mathrm{pH} 7.27, \mathrm{pCO2:45} \mathrm{mmHg}$

- Chest X-ray: raised left hemi diaphragm with no consolidation

All other initial investigations were unremarkable. Multiple teams were involved in his intensive care admission, including Intensive Care, respiratory, gastroenterology, neurology, cardiology, oncology, hematology, infectious diseases, palliative care, and psychiatry.

Early intervention included an increase in the patient's regular pyridostigmine dosing and a neostigmine challenge which demonstrated no improvement. The patient's regular neurologist suggested that his current presentation was likely not related to underlying myasthenia gravis. It was noted that the patient may have had poor immune function due to his myasthenia treatment and thymic carcinoma that this may be contributing to a worsening of his aspergillosis infection. However, a subsequent CT chest showed improvement from 6 weeks earlier and unchanged malignant disease. The exclusion of a neurological or infectious cause for this presentation led to oncological involvement and cardiac investigations (Figure 1).

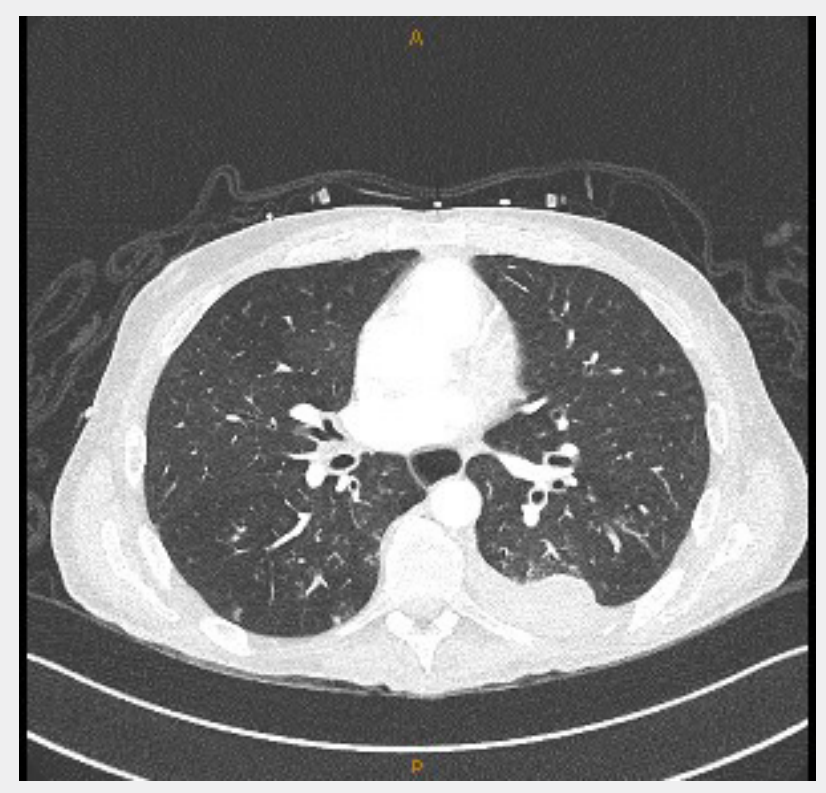

Figure 1: Transverse CT image showing the left sided nodular mass and minimal infective changes to the lung parenchyma.

An echocardiogram showed severe systolic dysfunction with large areas of hypokinesis and a Troponin I level ordered was $>9500 \mathrm{ng} / \mathrm{L}$ (normal $=<3 \mathrm{ng} / \mathrm{L}$ ). Furthermore, the patient suffered an episode of complete heart block which was managed 
with an isoprenaline infusion. An acute myocarditis triggered by the trial medication FN-1501 was suspected, and he was given Methylprednisolone 500mg per day for 3 days. The Troponin I level was monitored daily and was seen to come down in response to the steroid pulses, reaching a trough of $2411 \mathrm{ng} / \mathrm{L}$ on the third day of treatment. However, this did not correlate with clinical improvement. Furthermore, repeat echocardiograms showed worsening systolic function. ECG findings were difficult to interpret due to underlying conduction abnormalities.

Given the complexity of this case and minimal improvement in condition, a multidisciplinary team meeting was held after one week of hospital admission. This was attended by consultant physicians from intensive care, respiratory, neurology and oncology. The meeting concluded that there were several contributing factors to the patient's functional decline, weakness and continued need for respiratory support including:

- Deconditioning as a result of poor oral intake and malignant disease

- $\quad$ Paraneoplastic syndromes

- $\quad$ Fatigability due to refractory myasthenia gravis

- Myocarditis secondary to recent cancer therapy, corticosteroids use or antifungal medication.

Subsequently, the management plan was to optimise nutrition, continue to treat myasthenia gravis (including plasma exchange and IVIG), repeat pulse steroids followed by oral prednisolone, and continuing to monitor side effects and wean respiratory support. End of life care discussions were also commenced with the patient, although he consistently expressed his wish to continue any treatment with the potential to prolonging his life.

Throughout his stay, many ends of life and 'goals of care' discussions were conducted with the patient by different specialists. He expressed his frustration at the number of these discussions as, despite his prognosis, he wished to undertake lifeprolonging/curative treatment. During a review by a psychiatrist, the patient outlined his belief that the multiple ends of life discussions from different specialists had a negative effect on his mood. Emotional lability from prolonged steroid management may also have contributed to the patient's feelings of hopelessness and anxiety. After 41 days of management in intensive care, he was transferred to a palliative care facility where, he died after a gastrointestinal hemorrhage.

\section{Discussion}

We have presented a complex and protracted case. For the purposes of this paper, this discussion will focus on key points including: CDK/TK inhibitor cardiotoxicity, multi-disciplinary team care within intensive care units and the role of end-of-life care discussions. There is an established link between the use of TKI drugs and myocarditis. A 2007 study which measured cardiac biomarkers (e.g. Troponin I) and LV function in patients with GIST receiving sunitinib (a TKI) showed a decrease in ejection fraction of $>15 \%$ in $18 \%$ of cases [6]. It is unclear whether CDK inhibitors have a direct cardiotoxic effect, but it has been postulated that their ability to inhibit cell cycles may affect cardiomyocyte differentiation during times of stress, leading to cardiac dysfunction [8]. Targeted cancer therapies may affect the heart in several ways, commonly through on-target toxicity as certain kinases are involved in the signaling pathways of both cancer cells and cardiomyocytes. Interference with these pathways can lead to an endoplasmic reticular stress response and an increase in caspase activation, which induces apoptosis [8]. The authors propose that the patient's penultimate ICU admission, where he was intubated for haemoptysis less than 7 days after his last dose of FN-5101, may have been a significant enough stressor to allow FN-1501 to interrupt the cardiomyocyte lifecycle. More research in this area is required to help predict those at risk of this outcome.

A number of studies show the effectiveness of corticosteroids in the management of inflammatory myocarditis and immune mediated myocarditis $[9,10]$. However, this improvement takes place over many weeks and prolonged corticosteroid use is known to cause skeletal myopathy and emotional lability $[9,11]$, key issues that needed to be balanced in this case given the patient's myasthenia gravis and functional decline. Furthermore, voriconazole has recently also been reported to cause myositis in patients being treated for invasive aspergillosis [12].

Common paraneoplastic syndromes in thymoma include myasthenia gravis, myositis, encephalitis, acquired neuromyotonia and Morvan's syndrome. These syndromes often respond well to immunological therapy and are routinely screened for in patients undergoing treatment for thymomas [3]. Given the association between new immunological therapies and cardiac complications discussed above, it may be useful to routinely monitor patients undergoing trials of these therapies for cadiovascular complications as well with biomarkers and imaging modalities such as echocardiogram and cardiac MRI.

Overlapping pharmacological factors and underlying disease states in this case meant that it was extremely important to involve multiple teams. However, this approach poses risks to the continuity of information the patient receives and to the effective discussion of difficult topics. Shared decision making and effective end of life care conversations are associated with medical treatment, which is more likely to align with patient wishes, and subsequently better patient quality of life $[13,14]$. However, confronting mortality and discussing death can also be acutely distressing to some patients [15]. In our case, end-oflife discussions were initiated by multiple physicians on multiple occasions. This may be seen in a positive light; however, our patient was initially resistant to, and quite distressed by, discussions of this nature. An inconsistency in approach and messaging from different physicians may have amplified the patient's feelings of frustration and anxiety. The importance of these discussions cannot be understated, but their effectiveness relies on the patient being prepared to participate, and also on consistent prognostic 


\section{Cancer Therapy \& Oncology International Journal}

information. In a case where there are many teams involved in the care of a patient, it might be beneficial to designate one team member or specialty to initiate end-of-life discussions in order to prevent provoking excessive anxiety.

\section{Learning Points}

- Further research into the association between $\mathrm{CDK}$ inhibitors and myocarditis should be considered, including predicting those at risk and considering treatment options

- Routine screening for cardiovascular complications of immunological therapies against kinases may be useful

- For complicated patients with multiple specialty units involved, one physician should be nominated to lead end of life discussions

\section{Conflict of Interest}

The authors declare that there are no conflicts of interest in this case, financial or otherwise.

\section{References}

1. Akaishi T, Suzuki Y, Imai T, Tsuda E, Minami N, Nagane Y, et al. Response to treatment of myasthenia gravis according to clinical subtype. BMC Neurol 16(1): 225.

2. Romi $F$ (2011) Thymoma in myasthenia gravis: from diagnosis to treatment. Autoimmune Dis. 2011: 474512.

3. Evoli A, Lancaster E (2014) Paraneoplastic disorders in thymoma patients. J Thorac Oncol. 9(9 Suppl 2): S143-7.

4. Malumbres M, Barbacid M (2009) Cell cycle, CDKs and cancer: a changing paradigm. Nat Rev Cancer 9(3): 153-166.

5. Wang Y, Zhi Y, Jin Q, Lu S, Lin G, et al. (2018) Discovery of 4 - ( 7 H-Pyrrolo [2,3 - d] pyrimidin- 4 -yl) a mino $)-\mathrm{N}-(4-((4-$ methylpiperazin-1-yl)methyl)p henyl)-1H-pyrazole-3-carboxamide
(FN-1501), an FLT3- and CDK-Kinase Inhibitor with Potentially High Efficiency against Acute Myelocytic Leukemia. J Med Chem 61(4): 1499-1518.

6. Chu TF, Rupnick MA, Kerkela R, Dallabrida SM, Zurakowski D, et al. (2007) Cardiotoxicity associated with tyrosine kinase inhibitor sunitinib. The Lancet 370(9604): 2011-2019.

7. Kerkela R, Grazette L, Yacobi R, Iliescu C, Patten R, et al. (2006) Cardiotoxicity of the cancer therapeutic agent imatinib mesylate. Nat Med 12(8): 908-916.

8. Cheng H, Force T (2010) Molecular mechanisms of cardiovascular toxicity of targeted cancer therapeutics. Circ Res 106(1): 21-34.

9. Allanore Y, Vignaux O, Arnaud L, Puechal X, Pavy S, et al. (2006) Effects of corticosteroids and immunosuppressors on idiopathic inflammatory myopathy related myocarditis evaluated by magnetic resonance imaging. Ann Rheum Dis 65(2): 249-252.

10. J W Mason, J B O'Connell, A Herskowitz, N R Rose, B M McManus, et al (1995) A Clinical Trial of Immunosuppressive Therapy for Myocarditis. N Engl J Med 333(5): 269-275.

11. Brown SK, David A Khan, Nejtek V (1999) The Psychiatric Side Effects of Corticosteroids. Annals of Allergy, Asthma, \& Immunology 83(6): 495-500.

12. Shanmugam VK, Matsumoto C, Pien E, Rosen J, Kumar P, et al. (2009) Voriconazole-associated myositis. J Clin Rheumatol 15(7): 350-353.

13. Detering KM, Hancock AD, Reade MC, Silvester W (2010) The impact of advance care planning on end of life care in elderly patients: randomised controlled trial. BMJ 340: c1345.

14. Alexi A Wright, Baohui Zhang, Alaka Ray, Jennifer W Mack, Elizabeth Trice, et al. (2008) Associations Between End-of-Life Discussions, Patient Mental Health, Medical Care Near Death, and Caregiver Bereavement Adjustment. JAMA 300(14): 1665-1673.

15. Quill T (2000) Initiating End-of-Life Discussions with Seriously Il Patients. The Journal of the American Medical Association 284(19).

Your next submission with Juniper Publishers will reach you the below assets

- Quality Editorial service

- Swift Peer Review

- Reprints availability

- E-prints Service

- Manuscript Podcast for convenient understanding

- Global attainment for your research

- Manuscript accessibility in different formats

( Pdf, E-pub, Full Text, Audio)

- Unceasing customer service

Track the below URL for one-step submission https://juniperpublishers.com/online-submission.php 